\title{
METAMORPHISM OF COSMIC DUST: DIAGNOSTIC INFRARED SIGNATURES
}

\author{
Joseph A. Nuth III \\ Astrochemistry Branch, Code 691 \\ NASA/Goddard Space Flight Center \\ Greenbelt, MD 20771
}

Introduction. Nucleation is a non-equilibrium process: the products of this process are seldom the most thermodynamically stable condensates but are instead those which form fastest. It should not be surprising that grains formed in a circumstellar outflow will undergo some degree of metamorphism if they are annealed or exposed to a chemically active reagent. As a consequence of this processing in the laboratory one observes a continuous increase in the strength of the silicate absorption band at 20 microns relative to the 10 micron feature. In Section 1 we show that this ratio can be used as an indicator of the relative age of silicate condensates. Metamorphism of refractory particles continues in the interstellar medium (ISM) where the driving forces are sputtering by cosmic ray particles, annealing by high energy photons and grain destruction in supemova generated shocks. Studies of the depletion of the elements from the gas phase of the ISM tell us that if grain destruction occurs with high efficiency, then there must be some mechanism by which grains can be formed in the ISM. Laboratory studies of such a process (Moore, Tanabe, and Nuth, Ap. J. (Lett) 373, L31-L34, 1990) have shown that the frequency of the - $\mathrm{SiH}$ stretch can be used as an indicator of the oxidation state of the silicon in such grains. Highly reduced grains exhibit an $\mathrm{SiH}$ absorption near $2100 \mathrm{~cm}^{-1}$ whereas highly oxidized silicates absorb near $2300 \mathrm{~cm}^{-1}$ : this point is discussed in Section 2.

\section{The "Age" of Silicate Grains is Indicated by the Relative Strengths of the $\mathbf{1 0}$ and 20 Micron Bands}

In the outflows around oxygen-rich stars the chemical environment is still quite reduced due to the high abundance of hydrogen. Consequently the vapors which eventually condense to form silicate grains consist of reduced species such as silicon monoxide ( $\mathrm{SiO})$, iron $(\mathrm{Fe})$ and magnesium $(\mathrm{Mg})$ metal: Excess oxygen is tied up as water or hydroxyl $(\mathrm{OH})$ rather than in combination with either Fe or $\mathrm{Mg}$. When refractory vapors become sufficiently supersaturated to condense, the processes of nucleation and grain growth occur quite rapidly - much too rapidly in most cases for equilibrium to be maintained. One result of rapid condensation is the production of "underoxidized" grains consisting primarily of $\mathrm{SiO}, \mathrm{Fe}$ and $\mathrm{Mg}$ plus some additional oxygen incorporated as the grain reacted with water vapor and $\mathrm{OH}$. Once the refractory vapors have all condensed onto the grain cores these cores will continue to oxidize until chemical equilibrium is achieved and stable, silicate minerals are formed. This sequence is outlined in the paper by Nuth and Hecht (Astrophys. Sp. Sci. 163, 79-94, 1990). 
Such a definitive chemical sequence has very specific observational consequences. First, the initial condensate will consist mostly of isolated $\mathrm{SiO}$ units together with iron and magnesium metal. Very little of the SiO will have polymerized and only a small fraction of the metal will have oxidized. Because metals are not infrared active, metallic species in refractory grains will not be directly detectable. Isolated $\mathrm{SiO}$ units will absorb strongly near 10 microns due to the $\mathrm{SiO}$ vibrational stretch, while only the more polymerized $\mathrm{SiO}_{\mathrm{x}}$ units absorb near 20 microns as a result of the $\mathrm{O}-\mathrm{Si}-\mathrm{O}$ bending vibration. Both $\mathrm{MgO}$ and $\mathrm{FeO}$ absorb near 20 microns, but since most $\mathrm{Mg}$ and $\mathrm{Fe}$ will have condensed as metal, these oxides will contribute very little to grain absorption in this bandpass. Freshly condensed grains will therefore absorb strongly near 10 microns and only weakly, if at all, near 20 microns.

As the grains "age", several chemical processes will occur which will affect the spectral properties of the dust. First, the grains will continue to react with oxygen in the ambient environment; this will produce more polymerized $\mathrm{SiO}_{\mathrm{x}}$ and will also increase the oxidized fraction of $\mathrm{Mg}$ and $\mathrm{Fe}$. Both of these processes will increase the grain opacity near 20 microns quite significantly, whereas the continued oxidation of the $\mathrm{SiO}$ to "SiO 2 " will increase the opacity near 10 microns by at most a factor of two. The ratio of the grain absorption near 10 microns compared to that at 20 microns should therefore be a monotonically decreasing function with time even if the intrinsic strength of the 10 micron feature increases due to the oxidation of SiO. This point was used by Stencel et al. (Ap. J. (Lett.) 350, L45-8, 1990) to construct a dust chronology for Mira variables.

\section{The "4.6 Micron" Feature is an Indicator of the Chemical State of Silicate Grains}

Silicate grains have been produced in the laboratory by two separate and very different techniques, both of which allow the incorporation of hydrogen into the structure of the grains. In the first experimental setup, ices of $\mathrm{SiH}_{4}+\mathrm{CH}_{4}, \mathrm{SiH}_{4}+\mathrm{NH}_{3}$ or $\mathrm{SiH}_{4}+\mathrm{H}_{2} \mathrm{O}$ were irradiated by $1 \mathrm{MeV}$ protons to a total dose of $\sim 10^{15}$ protons $/ \mathrm{cm}^{2}$ then slowly warmed to room temperature. In a carbon-rich grain, the $\mathrm{SiH}$ fundamental stretch occurs near $2110 \mathrm{~cm}^{-1}$ while in an oxygen-rich residue this feature appears as a triplet with components at $2140 \mathrm{~cm}^{-1}, 2187$ $\mathrm{cm}^{-1}$ and $2244 \mathrm{~cm}^{-1}$. The spectra of grains produced via the second experimental technique the combustion of an $\mathrm{SiH}_{4}+\mathrm{O}_{2}$ mixture in hydrogen at a variety of temperatures - follow a similar pattern. Combustion at higher temperature yields more complete oxidation of the silicate: grains produced at higher temperatures generally exhibit $\mathrm{SiH}$ absorption at higher energy since they are more oxygen-rich.

The $\mathrm{SiH}$ feature in an "SiC" grain gradually undergoes oxidation in air at room temperature: the position of the major peak shifts from $2110 \mathrm{~cm}^{-1}$ in the "reduced" grain to $2172 \mathrm{~cm}^{-1}$ in the more oxidized silicate while a shorter wavelength spectral component develops near $2230 \mathrm{~cm}^{-1}$. Exposure of grains produced via combustion at intermediate temperatures to air and/or water (oxidizers) results in the loss of all long wavelength components of the $\mathrm{SiH}$ stretch, a small change in the position of the main peak, and some loss of hydrogen from the grains. Exposure to vacuum at elevated temperature for long time periods results in the loss of most of the hydrogen from the grains. Therefore for grains in circumstellar or interstellar environments where the grain temperature might exceed $300 \mathrm{~K}$ for considerable times, the elimination of the $\mathrm{SiH}$ feature is possible if the rate of replenishment by ion-implantation does not exceed the rate at which hydrogen is thermally driven from the grains. However, as long as some SiH exists in the grain, the energy of the $\mathrm{SiH}$ fundamental will be indicative of the oxidation state of the silicon in the grains. 